\title{
On regular hyperbolic fibrations
}

\author{
Deirdre Luyckx \\ Department of Pure Mathematics and Computer Algebra \\ Ghent University \\ Krijgslaan 281, S25 \\ 9000 Gent \\ Belgium \\ dluyckx@cage.ugent. be
}

Joint work with Matthew Brown, Gary Ebert

A hyperbolic fibration of $\operatorname{PG}(3, q)$ is a collection of $q-1$ hyperbolic quadrics $Q_{i}^{+}(3, q), i=1,2, \ldots, q-1$, and two lines $L_{0}, L_{\infty}$ in $\mathrm{PG}(3, q)$ that partition the points of $\mathrm{PG}(3, q)$, see [1]. A hyperbolic fibration is called regular if the lines $L_{0}$ and $L_{\infty}$ form a conjugate (skew) pair with respect to each of the polarities associated with the $q-1$ hyperbolic quadrics $Q_{i}^{+}(3, q)$ of the fibration. A regular hyperbolic fibration agrees on $L_{0}$, respectively $L_{\infty}$, if the extension to $\operatorname{GF}\left(q^{2}\right)$ of each quadric $Q_{i}^{+}(3, q), i=1,2, \ldots, q-1$, meets $L_{0}$, respectively $L_{\infty}$, in the same pair of points $\{x, \bar{x}\}$ which are conjugate with respect to the extension $\mathrm{GF}\left(q^{2}\right)$ of $\mathrm{GF}(q)$.

In [2], Baker, Ebert and Penttila show (algebraically) that a regular hyperbolic fibration that agrees on one of its lines corresponds to a flock of a quadratic cone in $\mathrm{PG}(3, q)$ with one conic (plane) specified, and conversely. For this correspondence, a unified geometric construction will be given for all $q$. Further it will be explained that all hyperbolic fibrations that agree on one of their lines, are necessarily regular. If $q$ is even, an even stronger result holds: in this case it will be pointed out that all hyperbolic fibrations are regular.

\section{References}

[1] R. D. Baker, J. M. Dover, G. L. Ebert, and K. L. Wantz. Hyperbolic fibrations of PG(3,q). European J. Combin., 20(1):1-16, 1999.

[2] R. D. Baker, G. L. Ebert, and Tim Penttila. Hyperbolic fibrations and q-clans. Des. Codes Cryptogr., 34(2-3):295-305, 2005. 